\title{
Las mujeres nikkei en Perú
}

\author{
The Nikkei women in Peru
}

Amelia Morimoto Hayashi ${ }^{1}$

\section{RESUMEN}

El presente artículo es una aproximación al tema de la inmigración y comunidad de origen japonés en Perú desde la perspectiva de género, en el que se destaca especialmente el caso de las mujeres inmigrantes japonesas y de sus descendientes peruanas de origen japonés o "nikkei".

\section{Palabras clave}

Historia, inmigración, comunidad, cultura, mujeres japonesas, trabajo, deportes, cifras de población, personajes de origen japonés

\section{ABSTRACT}

This article is an approach to the topic of the immigration and the community of the Japanese origin in Peru from the gender perspective, in which stands out especially the case of immigrant women of Japanese and their descendants Peruvian Japanese origin or "Nikkei".

\section{Keywords}

History, immigration, community, culture, Japanese women, work, sports, population figures, characters of Japanese origin

\section{Traducción de palabras del idioma japonés:}

Nikkei: palabra del idioma japonés que designa a la cultura, población e individuos de origen japonés, establecidos o nacidos fuera del Japón. Esta denominación se utiliza en el continente americano desde la década de 1980 con relación a la población de origen japonés y la cultura de ese origen.

Nisei: hijo o hija de japoneses o japoneses de segunda generación.

Yobiyose imin: "inmigración por llamado de parientes, amigos".

Shashin kekkon: "matrimonio por intercambio de fotografías"

\section{INTRODUCCIÓN}

En este artículo, se destaca la presencia de la mujer de origen japonés a lo largo de la historia de los inmigrantes japoneses y sus descendientes. Un antecedente acerca del tema específico de las mujeres de la comunidad "nikkei" peruana es el artículo "Las ausentes en la foto: las mujeres "nikkei" en Perú". En tal artículo se observóy analizóla presencia pública de la mujer "nikkei" mediante una revisión de las fotografías de un álbum impreso sobre la comunidad japonesa en Perú, publicado en el año 1924. Para el presente artículo, dedicado al tema de las mujeres "nikkei", el análisis se aborda en base de los antecedentes históricos

Psicóloga por la URP; Diploma de estudios especializados en Antropología (DEA) PUCP; Magíster en historia por la UNMSM. Actualmente candidata a doctora en Psicología por la UNMSM. 
de la inmigración japonesa a Perú en general y de las mujeres japonesas en Perú de manera específica; asimismo, de las estadísticas sobre la población de origen japonés, producto de los dos censos realizados sobre ella (en los años 1966 y 1989), de publicaciones (en revistas especialmente) de la comunidad "nikkei" y del caso específico de una mujer líder dentro de la comunidad "nikkei" peruana, además de los testimonios de mujeres "nikkei", recolectados a través de entrevistas formales e informales en las décadas de 1980 y 1990.

\section{LA INMIGRACIÓN DE MUJERES JAPONESAS A PERÚ}

El arribo de mujeres japonesas a Perú se produjo a principios del siglo $\mathrm{XX}$, años después del inicio de la inmigración de varones japoneses en 1899, durante la etapa denominada -en japonés- "yobiyose imin" o inmigración "por llamado de parientes o amigos" de los primeros inmigrantes. En tal período se permitió el ingreso de japoneses a Perú por solicitud de los inmigrantes de tal origen más antiguos; las mujeres inmigrantes eran generalmente esposas y madres de los hijos de los inmigrantes que habían arribado previamente. En otros casos, se trataba de mujeres japonesas solicitadas para el matrimonio con varones japoneses solteros, residentes en Perú. La inserción de estas mujeres japonesas fue esencial para la constitución de la comunidad "nikkei" en Perú; su presencia y rol a nivel público, sin embargo, permaneció generalmente limitado, sobre todo al interior de la estructura comunitaria.

Las mujeres japonesas arribaron por primera vez a Perú como parte del segundo contingente de inmigrantes japoneses en el año 1903, por exigencia de las haciendas agrícolas empleadoras, las que establecieron una nueva cláusula para el contrato de varones casados de manera exclusiva. Las razones manifestadas para tal cambio se relacionaron con las dificultades experimentadas con el primer grupo de inmigrantes, arribado a Perú en 1899 y compuesto por 790 varones. Según un documento de una hacienda agrícola de la costa peruana:
"Los juegos de azar, la ebriedad y el adulterio fueron comunes (...) El porcentaje de adulterio fue elevado debido a que el número de hombres casados era pequeño (menos de 70 de los 800 individuos japoneses eran casados). A manera de remedio, eran traídas prostitutas desde los pueblos. ...Considerando que la escasez de mujeres condujo a los males arriba mencionados..." (Fuente: Documento del Archivo del Fuero Agrario, en Morimoto, 1979)

El rol de las mujeres japonesas en Perú, por otro lado, no estuvo circunscrito a la procreación y asuntos domésticos; su presencia fue crucial en el trabajo e ingreso familiar y -en algunos casos- en la acumulación de recursos y progreso económico de las familias, tanto en la agricultura como asalariadas, "yanaconas" o agricultoras libres, como en la actividad urbana, en los pequeños establecimientos comerciales, como bodegas o pulperías. Para los hacendados de la costa peruana y para las compañías de inmigración japonesas, la presencia de mujeres y consecuente formación de familias, así como el ingreso a Perú de familias de japoneses, permitirían una mayor disciplina y productividad en el trabajo. Para fomentar el ingreso de mujeres japonesas, los hacendados agrícolas ofrecieron a las compañías de inmigración japonesas una comisión mayor: de 30 yenes por cada mujer japonesa y 20 por cada hombre (la comisión anterior era de 25 por cada varón). Sin embargo, hasta fines del período de inmigración de japoneses por contrato (1899 - 1923) ingresaron solo 2,145 mujeres y 226 niños, frente a la cifra mayoritaria de 15,887 varones adultos.

Es recién a partir de la segunda década del siglo $\mathrm{XX}$ que empezaron a llegar mujeres japonesas, bajo la modalidad denominada "matrimonio por intercambio de fotografías" ("Shashin kekkon", en japonés), además de familias completas o de mujeres e hijos de los japoneses casados y establecidos previamente en Perú. Al igual que los casos de los inmigrantes japoneses en otros países del continente americano, los varones solteros en Perú encargaban buscar esposas a las agencias japonesas de inmigración o a 
parientes y amistades en sus propias localidades de procedencia, proceso que generalmente se iniciaba con el intercambio de fotografías entre los varones y damas. Tal práctica para la concertación de matrimonios, sin embargo, estuvo limitada para quienes habían logrado acumular alguna pequeña fortuna o por lo menos alguna estabilidad económica, debido al costo que implicaba el traer a las esposas desde Japón. La unión con mujeres peruanas, por otro lado, ya se había iniciado, sobre todo en aquellas provincias peruanas en donde la población japonesa era más pequeña y en donde sus miembros establecieron mayor contacto $y$ se integraron a las poblaciones locales.

Las mujeres inmigrantes japonesas laboraban junto con sus esposos en establecimientos propios, como cafetines, restaurantes $y$ otros de mayor envergadura, como bazares. Paralelamente a tales responsabilidades laborales, la mayoría de las mujeres de la generación inmigrante estuvo también a cargo de la crianza y educación de, generalmente, varios hijos y de las labores del hogar. Salvo en casos aislados, la presencia de la mujer "issei" (primera generación o inmigrante) o "nikkei" (descendientes de japoneses en el exterior) exclusivamente dentro de la esfera doméstica fue posterior, recién a mediados del siglo XX.

\section{LAS CIFRAS DE POBLACIÓN "NIKKEI" POR GÉNERO}

La disparidad en las cifras hombres/mujeres, que fue notoria en la generación "issei" o de inmigrantes, fue desapareciendo con el nacimiento de nuevas generaciones. Los resultados de los censos específicos sobre la población "nikkei", realizados en los años 1966 y 1989, muestran una casi paridad numérica entre géneros. El censo sobre la población "nikkei" de 1966 arrojó la cifra de 32,002 individuos pertenecientes a cuatro generaciones; las más numerosas fueron las de los hijos de los inmigrantes o "nisei", y los "sansei" (tercera generación, nietos de los inmigrantes), seguidas por la generación de los inmigrantes o "issei" (primera generación, inmigrante) y la cuarta generación o "yonsei" (bisnietos de los inmigrantes). Las cifras porcentuales por género fueron: $48.29 \%$ (ó $15,452$ ) mujeres y $51.71 \%$ (ó 16,550$)$ varones (Morimoto, 1979, p.72).

El segundo censo "nikkei", realizado en 1989, dio como resultado la cifra total de 45,644 individuos, $49.2 \%$ o 22,485 varones y $50.8 \%$ o 23,137 mujeres; invirtiéndose los porcentajes por género con relación al censo anterior, de 1966, es decir, el segundo censo reveló, por vez primera en la historia de esta comunidad, una cifra ligeramente superior de mujeres "nikkei" frente a la de varones. Por otro lado, las generaciones más numerosas, según este último censo "nikkei", fueron la "sansei" o tercera con el $45.8 \%$ y la "yonsei" o cuarta generación con el 13.5\% del total poblacional (Morimoto, 1991, pp. 46 y 47; 1999, p. 149 - 150). Vale decir, en este último censo se observó un cambio poblacional importante con el incremento de las cifras de mujeres frente a las de varones, y de las dos generaciones más jóvenes frente a las generaciones de los inmigrantes y de sus hijos ("issei" y "nisei").

\section{UNA MUJER LÍDER DENTRO DE LA COMUNIDAD PERUANO-JAPONESA}

ElenaMiyokoYoshidade Kohatsu (1929-1998) fue la más destacada líder de la organización de la comunidad peruano japonesa a lo largo de su historia, llegando a ser la única mujer presidenta (en el año 1991) de la "Asociación Peruano Japonesa", desde que fuera creada en el año 1917. Fue también presidenta de la "Asociación Femenina Peruano-Japonesa" (Fujinkai) entre los años 1971 - 1972 y 1978 - 1979, además de ser parte de los consejos directivos de otras instituciones, como de la "Asociación Emmanuel", del "Policlínico Peruano Japonés", de la "Asociación Urasenke de Perú y del "Centro Jinnai" (Programa para ancianos de la comunidad), de la "Fundación del Museo Conmemorativo de la Inmigración Japonesa en el Perú", entre otros. Como semblanza, refiere uno de sus hijos: "...muchas personas tienen presente el recuerdo de mi progenitora y de su acertada forma de tomar decisiones.... lo que hacía Elena Kohatsu era simplemente utilizar el sentido común y tener el valor de aplicarlo". ${ }^{2}$

2 http://www.perushimpo.com/noticias.php?idp=2708\#sthash.SikjUG0k.dpuf 
Tal fue una acertada definición de las características más resaltantes de la señora Kohatsu, quien, desenvolviéndose en un medio en que el patriarcado fue característica fundamental desde sus inicios y en que las primeras generaciones de mujeres constituían su más fiel soporte, tuvo, precisamente, el valor de demostrar sus capacidades intelectuales y personales con serenidad y firmeza al interior de la organización comunitaria, cualidades por las que su liderazgo fuera reconocido $y$ apreciado.

\section{LOS "NIKKEI" EN LOS DEPORTES NACIONALES}

La práctica de deportes -varios de los cuales fueron traídos y desarrollados en Perú por los inmigrantes japoneses, como el "sumó" y el béisbol- fue parte de la vida cotidiana de los inmigrantes japoneses, desde los primeros años de trabajo en las haciendas, y fue inculcada a sus descendientes no solo a través del ejemplo, sino por el estímulo de los eventos de competición al interior de la comunidad y la construcción de espacios físicos para su práctica.

En la década de 1950, fue inaugurado el "Estadio La Unión", en el distrito de Pueblo Libre en Lima, por la comunidad peruanojaponesa, el que -desde entonces- congrega a miles de personas, tanto para la práctica cotidiana de distintos deportes -como fútbol, voleibol, béisbol, tenis, natación y atletismo, entre otros- como para la participación en competencias deportivas. Con el estadio nacieron también numerosos clubes deportivos, de cuyas filas surgieron varios personajes "nikkei" destacados en la actividad deportiva nacional. Algunos de ellos fueron:

Olga Azato, quien integró la Selección Nacional de Voleibol que conquistó numerosas veces el título de Campeona Sudamericana, recibiendo por ello los "laureles deportivos".

José Teruya obtuvo el Campeonato Latinoamericano de lucha libre, en la "modalidad libre" y "lucha grecorromana", categoría "mosca", en 1970. En 1976 recibió los "laureles deportivos".
Isabel Onchi representó a Perú en el Campeonato Mundial de bolos (bowling), realizado en los Estados Unidos en los años 1971 y 1976. Recibió los laureles deportivos por haber obtenido el trofeo máximo en el Campeonato Sudamericano de Bowling, en Buenos Aires.

Gladys Moriyama y María Guibu obtuvieron el campeonato en tenis de mesa en los Juegos Bolivarianos de Quito y en el XV Campeonato Sudamericano de Buenos Aires, en la categoría dobles damas. Individualmente, Moriyama representó a Perú en numerosos eventos internacionales y obtuvo los laureles deportivos en los años 1968, 1971 y 1972; fue también campeona sudamericana de tenis de mesa en equipos. Guibu obtuvo los laureles deportivos en el año 1972.

Humberto Suguimitsu, uno de los nombres más populares en la historia del deporte nacional, fue dos veces subcampeón mundial de billar, en eventos realizados en Lima en 1966 y en Las Vegas -Estados Unidos en 1970. Fue asimismo cuatro veces campeón sudamericano de billar y recibió los laureles deportivos en los años 1968 y en 1976 con el grado de Gran Cruz.

Erika Hayashida obtuvo varios títulos nacionales $e$ internacionales en golf, $y$ se convirtió en profesional de tal deporte a nivel internacional. Igualmente, el joven Akio Tamashiro obtuvo el máximo título en el Campeonato Panamericano de Karate, modalidad Kata, cuatro veces. En fútbol profesional han sido también reconocidos - entre otros - los talentos de Jorge Hirano, "Pedrito" Ruiz y, en los últimos años, José "chino" Pereda y Ernesto Arakaki, todos ellos descendientes de japoneses.

Los "nisei" (hijos de japoneses) se convirtieron también en promotores y dirigentes nacionales en el deporte. María Guibu fue directora de la sección tenis de mesa del Instituto de Recreación y Deportes (INRED). Gerardo Maruy, reconocido dirigente y deportista, fue varias veces presidente de la Federación Nacional de Béisbol y presidente del Instituto Peruano del Deporte (IPD). 
A esa generación de "nisei" deportistas le sucedió otra compuesta por jóvenes, hijos $y$ nietos de los inmigrantes, destacados en distintas disciplinas deportivas. En 1979, la selección nacional infantil de béisbol -integrada mayoritariamente por descendientes de japoneses- obtuvo el subcampeonato sudamericano en evento realizado en Lima; por ello recibió los laureles deportivos.

\section{LOS ARTISTAS E INTELECTUALES NIKKEI}

Uno de los aportes mayores de la inmigración japonesa en Perú se encuentra en el campo de la cultura, tanto por la presencia numéricamente importante de descendientes de japoneses en ella como por la calidad y significado de la obra de muchos de ellos.

En el campo de la cultura, por la vigencia de sus obras a través de varias décadas y por el reconocimiento unánime de la crítica especializada, Tilsa Tsuchiya en artes plásticas y José Watanabe en poesía son los personajes y nombres sobresalientes entre los descendientes de japoneses, sobre todo por su trascendencia más allá de las fronteras nacionales.

Dentro de las artes plásticas -pintura y esculturason numerosos los apellidos japoneses, como los de los consagrados artistas Arturo Kubotta y Venancio Shinki. Son también actualmente reconocidos los artistas plásticos nacidos en la posguerra: Eduardo Tokeshi, Jaime Higa, Oswaldo Higuchi y Jorge Oka, en pintura; Carlos Runcie Tanaka, en cerámica; así como los jóvenes artistas Aldo Shiroma, Hansel Sato, Haroldo Higa y Erika Nakasone, entre otros.

En el campo de la fotografía y en los inicios de la cinematografía en Perú, la obra de Eulogio Nishiyama ha permanecido como valioso aporte. En la cinematografía, como guionista, el poeta José Watanabe obtuvo también un amplio reconocimiento, tanto por el volumen de su producción como por su calidad.

En literatura, ha sido reconocido por la crítica el aporte de Augusto Higa en la narrativa. Asimismo, Doris Moromisato tiene una presencia reconocida entre las poetas nacionales.

La danza clásica en Perú - por otro ladoha contado con varias artistas y maestras de origen japonés, como Diana Kané, Hilda Nagasaki y Olga Shimazaki, quien no solo tuvo una reconocida trayectoria como figura del ballet, sino que su aporte en el mismo continuó como directora del Ballet Nacional.

La música popular en Perú ha contado también con varios "nisei", (hijos de japoneses), cuyos aportes en las distintas expresiones culturales han sido reflejo de la heterogeneidad de mundos en que se han desenvuelto los descendientes de japoneses y sus antecesores. En la composición musical, la prolífica y difundida producción de Luis Abelardo Takahashi Núñez es parte importante del acervo en música criolla nacional.

En la interpretación de música andina, ha sido también destacada la presencia de dos cantantes "nisei": Angélica Harada, "Princesita de Yungay" y Juan Makino Tori, "El Samurai del Huayno", cuyas trayectorias se desarrollaron especialmente en los distintos "coliseos" que congregaban a multitudinarias audiencias de origen provinciano en la ciudad de Lima.

Además de los aportes de los artistas y escritores, ha sido también significativo el aporte de los "nisei" en el campo de la actividad académica y en la investigación científica y tecnológica, como los del ingeniero Julio Kuroiwa en sismología y del doctor Juan Takano en oncología. 


\section{REFERENCIAS}

Morimoto, Amelia. 1979. Los inmigrantes japoneses en el Perú. Lima: Universidad Nacional Agraria, Taller de Estudios Andinos. Lima

1981. "Población de origen japonés en el Perú: Investigaciones y Bibliografía", en: Primer Seminario sobre Poblaciones inmigrantes. Lima, Consejo Nacional de Ciencia y Tecnología, año 1987, Tomo I, pp. 105 - 140 (autora y Editora). Lima

1989. "Inmigración y Comunidad de origen japonés en el Perú", en Europa, Asia y África en América Latina. México, UNESCO - Ed. Siglo XXI, 1989; pp. 291 - 322 (autora y Editora). Lima

1992. "Peru no Nihonjin Imin". Nippon Hyoronsha (Traducción al japonés por Sakimori Kon). Tokio

1999. "Los japoneses y sus descendientes en el Perú". Lima: Fondo Editorial del Congreso del Perú; 255 pp.

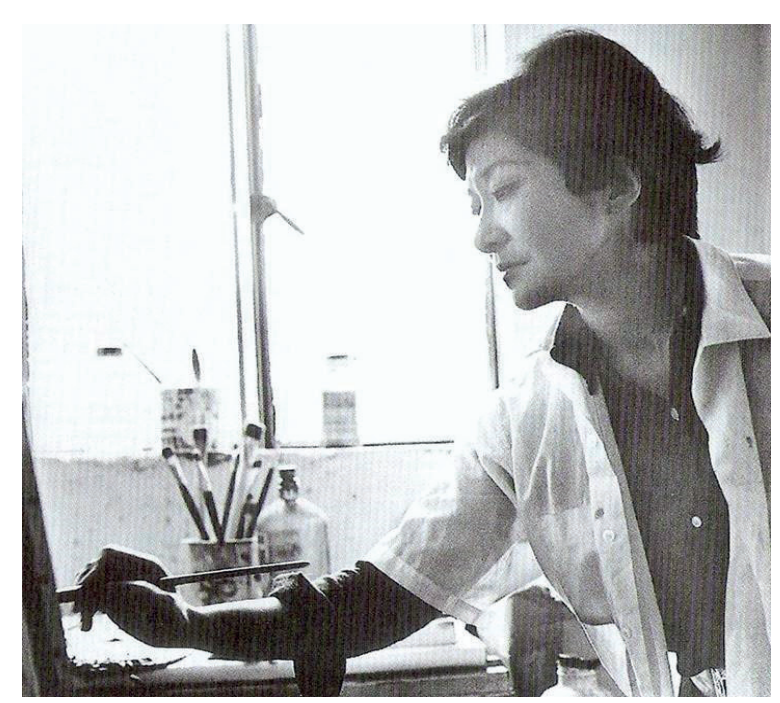

Artista plástica TILSA TSUCHIYA CASTILLO, Supe 1928 - Lima, 1984 (+)

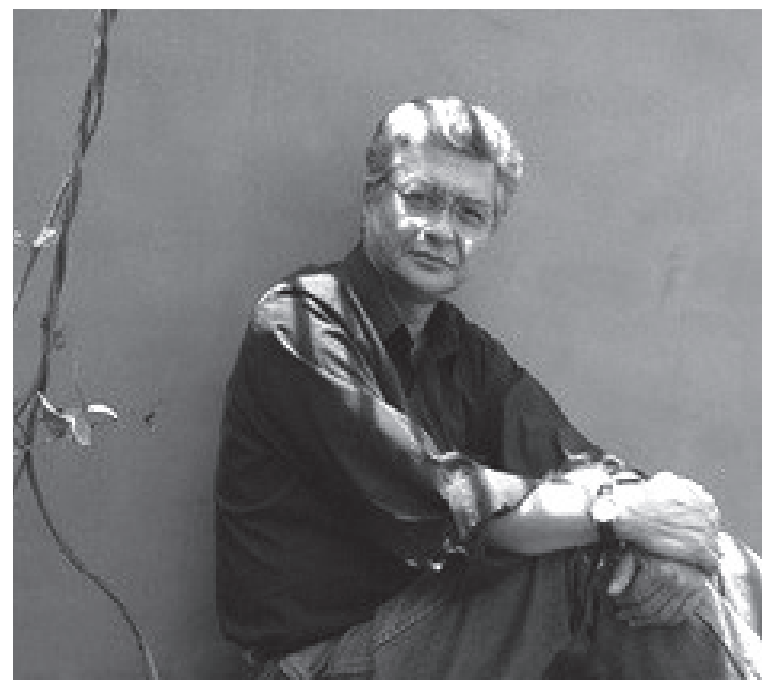

Poeta y cineasta JOSÉ WATANABE VARAS, Laredo 1946 - Lima 2007 (+) (Fotografía: Paul Vallejos)

Fecha de recepción: 13-05-18.

Fecha de aceptación: 30-05-18. 